\title{
First Detection of Penicillium fellutanum from Stored Rice in Korea
}

\author{
Ji Yeon $\mathrm{Oh}^{1}$, Mee Kyung Sang ${ }^{1,2}$, Hojoung Lee ${ }^{3}$, Mun Il Ryoo ${ }^{4}$ and Ki Deok Kim ${ }^{1 *}$ \\ ${ }^{1}$ Laboratory of Plant Disease and Biocontrol, College of Life Sciences and Biotechnology, \\ Korea University, Seoul 136-713, Korea \\ ${ }^{2}$ Institute of Life Science and Natural Resources, Korea University, Seoul 136-713, Korea \\ ${ }^{3}$ Laboratory of Applied Plant Stress Biology, College of Life Sciences and Biotechnology, \\ Korea University, Seoul 136-713, Korea \\ ${ }^{4}$ Division of Environmental Science and Ecological Engineering, Korea University, Seoul 136-713, Korea \\ (Received on June 18, 2011; Revised on June 21, 2011; Accepted on June 21, 2011)
}

\begin{abstract}
A representative isolate KU53 of the predominant Penicillium species was obtained from rice samples from rice processing complexes of National Agricultural Cooperative Federation in Korea. In this study, isolate KU53 was identified by its morphological and molecular characteristics. The macro- and microscopic characteristics of isolate KU53 were compared with the P. fellutanum reference isolate KCTC16913 on different media; isolate KU53 was generally identical to those of the reference isolate KCTC16913. In a molecular-based identification, the $\beta$-tubulin and translation elongation factor 1-alpha sequences of isolate KU53 was most closely related to those of $P$. fellutanum. Thus, isolate KU53 from stored rice could be identified as $P$. fellutanum, some isolates of which are known to produce mycotoxin-related metabolites. To our knowledge, this is the first detection of $P$. fellutanum from stored rice in Korea.
\end{abstract}

Keywords : Fungal identification, Penicillium fellutanum, Rice mycoflora, Stored rice

The species of the genus Penicillium are well known as one of the most serious grain contaminants due to production of mycotoxin (ochratoxins, patulin, and citrinin) and related fungal metabolites (alkaloids, amino acids, anthraquinoid, or azaphilone) (Bräse et al., 2009; Zhelifonova et al., 2010). Among these, certain isolates of $P$. fellutanum are reported to produce ergot alkaloids (agroclavine 1 and epoxyagroclavine 1) and diketopiperazine alkaloids (fellutanine $\mathrm{A}, \mathrm{B}, \mathrm{C}$, and D) (Bräse et al., 2009; Kozlovsky et al., 2000; Vinokurova et al., 2003). Ergot alkaloids exhibit various biological functions including antiherbivory and therapeutic effects (Clay, 1988; Fliger et al., 1997). These fungal isolates have been detected in sorghum, wheat, and corn grains in Argentina and other countries (Gonzlez et al., 1995, 1997); however, the isolates have not been recovered in stored rice in Korea.

In our previous studies (Oh et al., 2008a, b), we monitored the temporal changes of the microbial population and diversity in stored rice under controlled

*Corresponding author

Phone) +82-2-3290-3065, Fax) +82-2-925-1970

Email)kidkim@korea.ac.kr conditions. Consequently, we found that various Penicillium spp. existed in stored rice and $P$. islandicum, a mycotoxin producer, was the most predominant species. The population of this fungal species was dramatically increased up to $40-70 \%$ of the total fungal population in relation with increased relative humidity. In another studies (Oh et al., 2007, 2010), we observed various fungal populations and diversity existed in unhulled and white rice samples collected from rice processing complexes of National Agricultural Cooperative Federation of various regions in Korea. Among the observed fungi, which included Aspergillus candidus, A. flavus, and A. fumigatus, Penicillium species were predominant (Oh et al., 2007, 2010); thus, one (representative isolate KU53) of the most frequently observed Penicillium spp. was selected and identified by its morphological and molecular characteristics.

Morphological characteristics of isolate KU53 were examined on different media: czapek yeast agar (CYA), czapek yeast with $5 \% \mathrm{NaCl}$ (CYAS) agar, czapek dox agar (CZ), malt extract agar (MEA), dichloran 18\% glycerol agar (DG18), yeast extract sucrose agar (YES), $25 \%$ glycerol nitrate agar (G25N), creatine sucrose agar (CREA) (Frisvad and Samson, 2004; Pitt and Hocking, 1999). The characteristics of the test isolate 
were compared to those of the $P$. fellutanum reference isolate KCTC16913 from the Korean Collection for Type Cultures (KCTC), Daejeon, Korea, as well as to those described by Pitt (2000). In these tests, $10 \mu \mathrm{l}$ of spore suspensions of isolate KU53 or KCTC16913 amended with $0.03 \%$ Tween 20 were inoculated on each medium at three spots at equivalent distance. These inoculated plates were grown at $25^{\circ} \mathrm{C}$ for 7 days; to compare growth rates, the plates of CYA medium were grown at 15,25 , and $30^{\circ} \mathrm{C}$, respectively. We examined the macro-morphological characteristics on each medium including diameters, overall color, colors of conidia, reverse colors, as well as production of sclerotia, exudates, and soluble pigments. Ehrlich reactions were also conducted to detect indole metabolites produced by the isolates KU53 and KCTC16913 as described by Lund (1995).

To examine micro-morphological characteristics, isolate KU53 and reference isolate KCTC16913 were grown on MEA at $25^{\circ} \mathrm{C}$ for 7 days. Micro-morphological characteristics such as the shape and length of conidia, roughness of conidial walls, lengths of phialides, metulae, and stipes, conidiophores, branching patterns, appressedness, existence of ascospores or cleistothecia were observed using a microscope $(\times 1000)$. The experiment was conducted twice with three replicates each and data were analyzed using SAS version 9.1.3 (SAS Institute, Cary, NC, USA).

For molecular identification of isolate KU53, mycelial DNA was extracted from cultures grown in potato dextrose broth at $28^{\circ} \mathrm{C}$ for 7 days; DNA was extracted by the modified method of Boom et al. (1990). The primer pairs used for Penicillium species amplified $\beta$ tubulin (BT2a: 5'-GGT AAC CAA ATC GGT GCT GCT TTC-3', Bt2b: 5'-ACC CTC AGT GTA GTG ACC CTT GGC-3') and translation elongation factor 1-alpha (EF-1 $\alpha)$ (EF6: 5'-CTT-STY CCA RCC CTT GTA CCA-3', EF1d: 5'-GGC CAC GTC GAT TCC GG-3'). Polymerase chain reaction (PCR) conditions were as follows: $10 \mu \mathrm{l}$ of each reaction mixture containing $1 \mu \mathrm{l}(10 \mathrm{ng} / \mu \mathrm{l})$ of template DNA, $1 \mu \mathrm{l}(20 \mathrm{ng} / \mu \mathrm{l})$ of forward primer, $1 \mu \mathrm{l}(20 \mathrm{ng} / \mu \mathrm{l})$ of reverse primer, $1 \mu \mathrm{l}$ of $2.5 \mathrm{mM} \mathrm{dNTP}, 1 \mu \mathrm{l}$ of $10 \times$ reaction buffer, $0.1 \mu \mathrm{l}$ ( 5 unit/ $\mu \mathrm{l}$ ) of Taq, and $4.9 \mu \mathrm{l}$ of distilled water. Cycling conditions were as follows: $95^{\circ} \mathrm{C}$ for $4 \mathrm{~min}$ for one cycle followed by 30 cycles of DNA denaturation at $95^{\circ} \mathrm{C}$ for $30 \mathrm{sec}$, primer annealing at $60^{\circ} \mathrm{C}$ for $1 \mathrm{~min}$, and DNA extension at $72^{\circ} \mathrm{C}$ for $1 \mathrm{~min}$, and a final extension cycle at $72^{\circ} \mathrm{C}$ for $7 \mathrm{~min}$. PCR products were electrophoresed on $2 \%$ agarose gel; product bands were excised from the gel, extracted with MEGA-spin ${ }^{\mathrm{TM}}$ (iNtRON Biotechnology, Seoul, Korea), and ligated into pGEM ${ }^{\circledR}$-T-Easy Vector (Cat.\# A1360, Promega, Madison, USA). Cloned plasmids were transformed into Escherichia coli DH5 $\alpha$ by chemical methods. The resulting DNA constructs were purified using DNA$\operatorname{spin}^{\mathrm{TM}}$ (iNtRON Biotechnology, Seoul, Korea) and checked by digestion with the restriction endonuclease HindIII. The purified plasmid DNA was also sequenced with M13 universal primer (5'-GTT TTC CCA GTC ACG AC-3'). DNA sequences were compared to those of related Penicillium species using BLAST network services at the National Center for Biotechnology Information (NCBI) of the U.S. National Library of Medicine, Bethesda, MD, USA. A phylogenetic tree was constructed with the neighbor-joining method using Molecular Evolutionary Genetics Analysis (MEGA) version 3.1 program (The Biodesign Institute, Tempe, AZ, USA), and bootstrap analysis was also conducted using the same program. The $\beta$-tubulin and EF-1 $\alpha$ sequences of isolate KU53 have been deposited in GenBank under accession numbers FJ517585 and FJ517586, respectively.

Isolate KU53 was identified by comparing its macroand micro-morphological characteristics to those of reference isolate KCTC16913 and its description in the literature (Pitt, 2000) (Fig. 1 and Table 1). Isolate KU53 showed restricted growth and sporulation on CYA at $15^{\circ} \mathrm{C}(11-12 \mathrm{~mm})$ as observed in isolate KCTC16913 (10-11 mm) (Fig. 1). Colonies of isolate KU53 on CYA25 were dull turquoise and radially sulcate with heavy sporulation and thin white margin; reverse of the colony was radially sulcate and creamcolored with a green shade. Colonies of isolate KCTC16913 were quite similar to those of isolate KU53 except with less sporulation, a wide margin and a somewhat yellowish reverse color. The growth was 24-26 mm for isolate KU53, 23-25 mm for isolate KCTC16913, and 17-24 $\mathrm{mm}$ in the literature (Pitt, 2000). Colonies of isolate KU53 on CYA30 differed little from those on CYA25 but were dark-colored with a clear margin and somewhat slow growing (21-22 $\mathrm{mm}$ ); isolate KCTC16913 showed heavier sporulation and was more sulcate than isolate KU53. Colonies of isolate KU53 on CYAS were similar to those on CYA25 with a colony diameter of 23-26 mm. Isolate KU53 was similar to isolate KCTC16913 with the exception of slower growth (19-22 mm) and a dull margin in the reference isolate. Colonies of isolate KU53 on CZ differed little from those on CYA30 but grew slowly (18-19 mm). The isolate was also similar to isolate KCTC16913 on $\mathrm{CZ}$ except that the reference isolate exhibited slower growth (15-16 mm) and less sporulation. Colonies of isolate KU53 on MEA were 

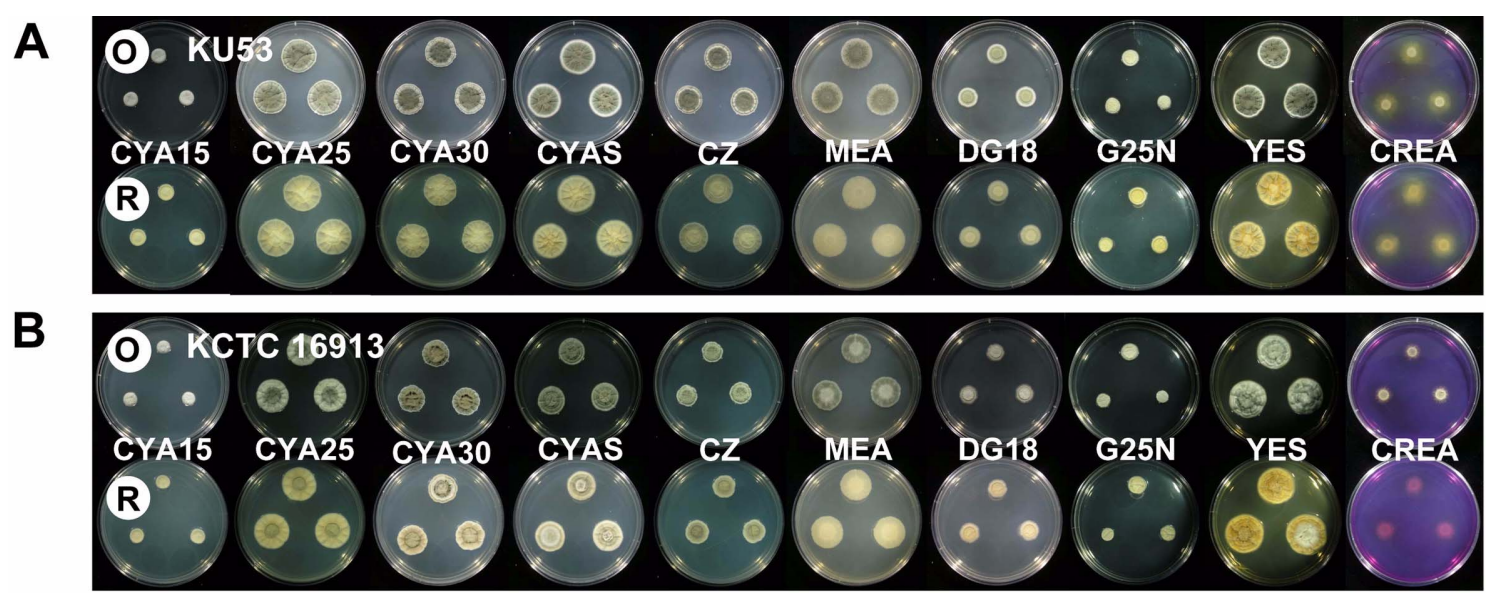

Fig. 1. Morphologies of (A) the Penicillium fellutanum isolate KU53 and (B) the P. fellutanum reference isolate KCTC16913 on different media: czapek yeast extract agar grown at $15^{\circ} \mathrm{C}$ (CYA15), CYA at $25^{\circ} \mathrm{C}$ (CYA25), CYA at $30^{\circ} \mathrm{C}$ (CYA30), czapek yeast extract agar with $5 \% \mathrm{NaCl}$ (CYAS), czapek dox agar (CZ), malt extract agar (MEA), dichloran 18\% glycerol agar (DG18), 25\% glycerol nitrate agar (G25N), yeast extract sucrose agar (YES), and creatine sucrose agar (CREA). O, Obverse; R, reverse of the plate.

Table 1. Characteristics of Penicillium fellutanum isolate KU53 and reference isolate KCTC16913 in comparison with characteristics described by Pitt (2000)

\begin{tabular}{|c|c|c|c|c|}
\hline \multicolumn{2}{|c|}{ Characteristics } & $\begin{array}{l}\text { Present isolate } \\
\text { KU53 }\end{array}$ & $\begin{array}{c}\text { Reference isolate } \\
\text { KCTC16913 }\end{array}$ & Pitt (2000) \\
\hline \multicolumn{5}{|l|}{ Quantitative characters } \\
\hline \multirow[t]{12}{*}{ Microscopic features $^{\mathrm{a}}$} & Conidia $^{\mathrm{b}}$ & & & \\
\hline & Shape & Subglobose to ellipsoidal & Subglobose to ellipsoidal & Ellipsoidal \\
\hline & Length & $2.5-3.6 \mu \mathrm{m}$ & $2.6-3.6 \mu \mathrm{m}$ & $2.5-3.2 \mu \mathrm{m}$ \\
\hline & Roughness & Smooth & Smooth & Smooth or finely roughened \\
\hline & Phialide length $^{c}$ & $6-9 \mu \mathrm{m}$ & $5-9 \mu \mathrm{m}$ & $5-10 \mu \mathrm{m}$ \\
\hline & Metulae length & $14-19 \mu \mathrm{m}$ & $14-19 \mu \mathrm{m}$ & $10-30 \mu \mathrm{m}$ \\
\hline & Stipe & & & \\
\hline & Length & $31-40 \mu \mathrm{m}$ & $30-40 \mu \mathrm{m}$ & $-^{\mathrm{d}}$ \\
\hline & Roughness & Smooth & Smooth & Smooth \\
\hline & Conidiophore & & & \\
\hline & Branching pattern & Monoverticillate & Monoverticillate & Monoverticillate \\
\hline & Appressedness & Appressed & Appressed & - \\
\hline \multirow{3}{*}{$\begin{array}{l}\text { Ecophysiological } \\
\text { characteristics }^{\mathrm{e}}\end{array}$} & CYA30 / CYA25 & 0.85 & 0.86 & - \\
\hline & CYA15 / CYA25 & 0.46 & 0.43 & - \\
\hline & CYAS / CYA25 & 0.98 & 0.85 & - \\
\hline Ehrlich reaction & & Yellow & Yellow & - \\
\hline \multicolumn{5}{|l|}{ Qualitative characters } \\
\hline \multirow{2}{*}{$\begin{array}{l}\text { Reaction on } \\
\text { creatine sucrose agar }\end{array}$} & Acid production & Moderate & Weak & - \\
\hline & Base production & None & None & - \\
\hline
\end{tabular}

${ }^{\mathrm{a}}$ All the features were observed and determined using a microscope $(\times 1,000)$.

${ }^{b}$ Lengths of conidia were determined from 50 observations.

${ }^{c}$ Lengths of phialide and metulae were determined from 30 observations and stipes were from 20 observations.

${ }^{\mathrm{d}}$ Not described.

${ }^{\mathrm{e}}$ Ratios of the diameter of fungal colonies on czapek yeast agar (CYA) media grown at 30,25 , and $15^{\circ} \mathrm{C}$ and czapek yeast sucrose agar with $5 \%$ $\mathrm{NaCl}(\mathrm{CYAS})$ grown at $25^{\circ} \mathrm{C}$. 
almost the same as those of isolate KCTC16913, both showing a dull turquoise color, granular, plane and cream reverse. Colony diameters were also similar: 22$24 \mathrm{~mm}$ for isolate KU53, 21-22 $\mathrm{mm}$ for isolate $\mathrm{KCTC} 16913$, and $14-18 \mathrm{~mm}$ in the literature (Pitt, 2000). The only difference was that isolate KCTC16913 remained less sporulated in the center. Colonies of isolate KU53 on DG18 were also similar to those of isolate KCTC16913, as both were slow growing (KU53=14-15 $\mathrm{mm}$ and KCTC16913=13-14 $\mathrm{mm}$ ) and centrally raised with a white margin. However, isolate KU53 produced dull green conidia while isolate KCTC16913 sporulated slightly. On G25N, both isolates KU53 and KCTC16913 were restricted in sporulation, but showed different reverse colors: yellow and cream, respectively. The diameters of isolates KU53 and KCTC16913 and the literature (Pitt, 2000) were 12-13, 11-12, and 12-16 mm, respectively. Colonies of isolate KU53 on YES were 25-31 $\mathrm{mm}$ in diameter, dull turquoise, and sulcate with a clear margin, and the reverse was yellow to cream with a green shade. Colonies of isolate KCTC16913 were quite similar to those of isolate KU53 except they were somewhat floccose with less sporulation. On CREA, isolate KU53 grew faster $(16-18 \mathrm{~mm})$ and produced more yellow pigment, indicating acid production than isolate KCTC16913 (13-14 mm). In all tested media, neither sclerotia nor exudates were produced (Fig. 1). In Ehrlich test, both isolates KU53 and KCTC16913 showed alkaloid production with yellow rings (Table 1).

Conidiophores of isolate KU53 and reference isolate KCTC16913 were borne from aerial hyphae, and were appressed and monoverticillate (Fig. 2A and Table 1); sometimes branch-like metulae developed at the lower part of the conidiophore (Fig. 2B and Table 1). The stipes of isolate KU53 were straight, smooth, and 31$40 \mu \mathrm{m}$ in length and did not differ from those of the

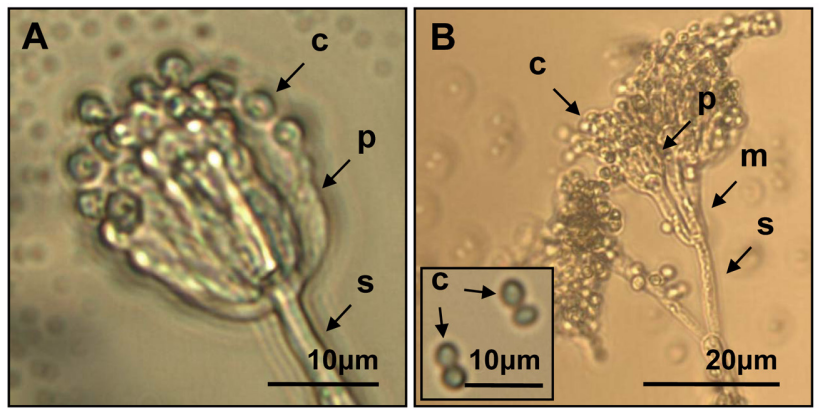

Fig. 2. (A) A monoverticillate penicillus and (B) conidiophores and conidia (close-up conidia in the inset) of the Penicillium fellutanum isolate KU53. c, conidia; m, metulae; p, phialide; s, stipes. reference isolate $(30-40 \mu \mathrm{m})$. Phialides of isolate KU53 were 6-9 $\mu \mathrm{m}$, parallel and flask-shaped, consisting of a cylindrical base covering the upper surface of the vesicles. Isolate KCTC16913 produced similar phialides of 5-9 $\mu \mathrm{m}$ in length as those described by Pitt (2000). Metulae of isolate KU53 and isolate KCTC16913 were smooth and 14-19 $\mu \mathrm{m}$ for both. Conidia of isolate KU53 were 2.5-3.6 $\mu \mathrm{m}$, subglobose to ellipsoidal with smooth-wall in loose columns and similar to those of the reference isolate $(2.6-3.6 \mu \mathrm{m})$ and those describe by Pitt (2000) (2.5-3.2 $\mu \mathrm{m})$ (Table 1$)$.

The phylogenetic tree based on partial $\beta$-tubulin sequence (478 bp) of isolate KU53 and other species belonging to genus Penicillium showed that isolate KU53 is most closely related to P. fellutanum (Fig. 3). The $\beta$-tubulin sequence of the test isolate showed the highest nucleotide identity (94\%) with P. fellutanum isolated from cork (EF198548 and EF198545). The partial EF-1 $\alpha$ sequence (480 bp) of isolate KU53 showed the highest nucleotide identities of 92 and $93 \%$ with $P$. fellutanum (AY741782) and $P$. charlesii (AY741756), respectively. Thus, phylogenetic placement of Penicillium species including isolate KU53 showed that $P$. fellutanum and $P$. charlesii are very closely related. $P$. fellutanum was formerly named $P$. charlesii or P. dierckxii (Pitt, 1973; Stolk and Samson, 1983). Since $P$. charlesii and $P$. fellutanum had similar characteristics such as growth rates, macro- and micromorphological characteristics, they were treated as synonyms (Pitt, 1973). Thus, isolate KU53 from stored rice in this study could be considered P. fellutanum.

Many researchers have studied Penicillium species associated with rice in Korea. Kim et al. (2005) isolated $P$. chrysogenum, $P$. citrinum, $P$. cyclopium, $P$. oxalicum, P. polonicum, P. purpurogenum, P. viridicatum, and $P$. chrysogenum from rice. Mheen et al. (1982) reported Penicillium spp. in stored rice, including $P$. atramentosum, $P$. chrysogenum, $P$. cyaneofulvum, $P$. notatum, and $P$. steckii. Park et al. (2005) also detected $P$. citrinum, $P$. islandicum, and $P$. verrucosum from polished rice. In addition to these reports on rice, Cho and Cheon (1962) found P. bioforme and P. brefeldianum from paddy field. Likewise, there was a report on $P$. fellutanum from Nuruk, traditional Korean malt made from wheat (Jo and Lee, 1997). This species of $P$. fellutanum has been reported to be osmotolerant and xerotolerant (Park et al., 1998) and to have a narrow growth temperature range (Pitt, 2000). With these characteristics, the isolate KU53 tested in this study might produce alkaloid metabolites since a positive reaction was observed in the Ehrlich test. Taken together, these results indicated that the isolate KU53 


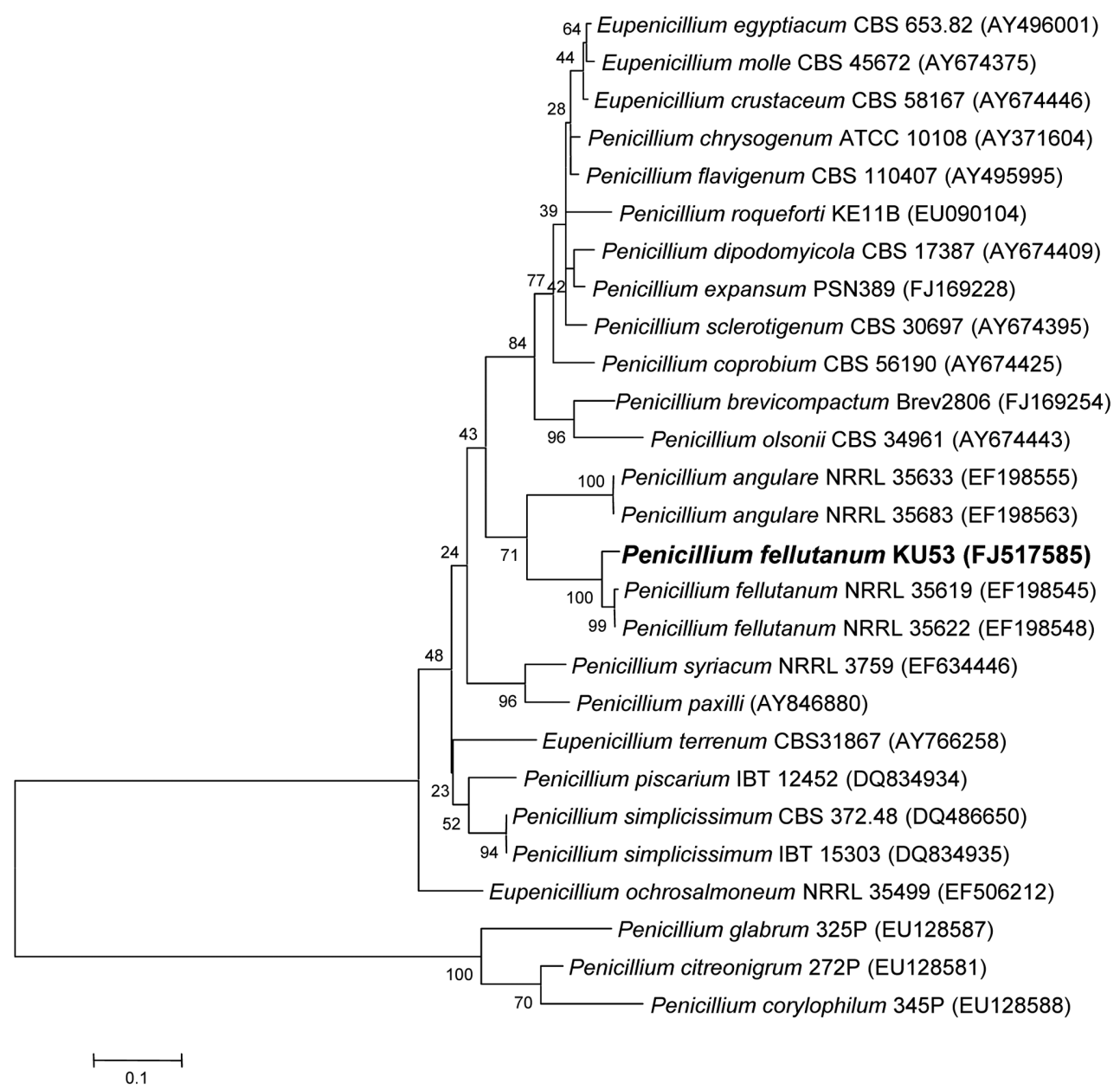

Fig. 3. A neighbor-joining tree of the Penicillium species relative to the $P$. fellutanum isolate KU53 based on phylogenetic analysis of $\beta$ tubulin sequence. Bootstrap values of 100 analyses are shown at the branching points and accession number of each species is placed in parentheses.

from stored rice could be identified as P. fellutanum, some isolates of which are known to produce mycotoxinrelated metabolites. To our knowledge, this is the first detection of $P$. fellutanum from stored rice in Korea.

\section{Acknowledgement}

This study was conducted with the support of "Specific Joint Agricultural Research-Promoting Projects (Project No. 20070101-033-025-001-03-11)", RDA, Suweon, Republic of Korea.

\section{References}

Boom, R., Sol, C. J. A., Salimans, M. M. M., Jansen, C. L., Wertheim-Van Dillen, P. M. E. and Van Der Noordaa, J. 1990. Rapid and simple method for purification of nucleic acids. $J$. Clin. Microbiol. 28: 495-503.
Bräse, S., Encinas, A., Keck, J. and Nising, C. F. 2009. Chemistry and biology of mycotoxins and related fungal metabolites. Chem. Rev. 109: 3903-3990.

Cho, J.-H. and Cheon, J.-K. 1962. Mycoflora of paddy field. Kor. J. Agr. Chem. 3: 17-18.

Clay, K. 1988. Fungal endophytes of grasses: a defensive mutualism between plants and fungi. Ecology 69: 10-16.

Fliger, M., Wirst, M. and Shelby, R. 1997. Ergot alkaloids: sources, structures and analytical methods. Folia Microbiol. 42: $3-30$.

Frisvad, J. C. and Samson, R. A. 2004. Polyphasic taxonomy of Penicillium subgenus Penicillium: A guide to identification of food and air-borne terverticillate Penicillia and their mycotoxins. Stud. Mycol. 49: 1-174.

González, H. H. L., Martínez, E. J. and Resnik, S. L. 1997. Fungi associated with sorghum grain from Argentina. Mycopathologia 139: 35-41.

González, H. H. L., Resnik, S. L., Boca, R. T. and Marasas, W. F. 
O. 1995. Mycoflora of Argentinean corn harvested in the main production area in 1990. Mycopathologia 130: 29-36.

Jo, G.-Y. and Lee, C.-W. 1997. Isolation and identification of the fungi from Nuruk. J. Korean Soc. Food Sci. Nutr. 26: 759766.

Kim, M. K., Hyun, I. H. and Kim, J. W. 2005. Identification of seed-borne Penicillium spp. on Gramineae crops based on morphological characteristics. Korean J. Mycol. 33: 81-85.

Kozlovsky, A. G., Vinokurova, N. G., Adanin, V. M., Burkhardt, G., Dahse, H.-M. and Gräfe, U. 2000. New diketopiperazine alkaloids from Penicillium fellutanum. J. Nat. Prod. 63: 698700.

Lund, F. 1995. Differentiating Penicillium species by detection of indole metabolites using a filter paper method. Lett. Appl. Microbiol. 20: 228-231.

Oh, J. Y., Jee, S. N., Nam, Y., Lee, H., Ryoo, M. I. and Kim, K. D. 2007. Populations of fungi and bacteria associated with samples of stored rice in Korea. Mycobiology 35: 36-38.

Oh, J. Y., Kim, E. N., Ryoo, M. I. and Kim, K. D. 2008 a. Morphological and molecular identification of Penicillium islandicum isolate KU101 from stored rice. Plant Pathol. J. 24: 469-473.

Oh, J. Y., Sang, M. K., Lee, S. Y., Ryoo, M. I. and Kim, K. D. 2008b. Temporal changes of fungal and bacterial populations in rice under indoor storage conditions. Plant Pathol. J. 24: $74-79$.

Oh, J. Y., Sang, M. K., Oh, J.-E., Lee, H., Ryoo, M. I. and Kim, K.
D. 2010. Microbial population, aflatoxin contamination and predominant Aspergillus species in Korean stored rice. Plant Pathol. J. 26: 121-129.

Park, J. W., Choi, S., Hwang, H. and Kim, Y. 2005. Fungal mycoflora and mycotoxins in Korean polished rice destined for humans. Int. J. Food Microbiol. 103: 305-314.

Park, Y.-I. and Gander, J. E. 1998. Choline derivatives involved in osmotolerance of Penicillium fellutanum. Appl. Environ. Microbiol. 64: 273-278.

Pitt, J. I. 1973. An appraisal of identification methods for Penicillium species: novel taxonomic criteria based on temperature and water relations. Mycologia 65: 1135-1157.

Pitt, J. I. 2000. A laboratory guide to common Penicillium species. 3rd ed. Food Science Australia, North Ryde, Australia. 197 pp.

Pitt, J. I. and Hocking, A. D. 1999. Fungi and food spoilage. 2nd ed. Aspen Publications, Gaithersburg, USA. 413 pp.

Stolk, A. C. and Samson, R. A. 1983. The ascomycete genus Eupenicillium and related Penicillium anamorphs. Stud. Mycol. 23: 1-149.

Vinokurova, N. G., Boichenko, L. V. and Arinbasarov, M. U. 2003. Production of alkaloids by fungi of the genus Penicillium grown on wheat grain. Appl. Biochem. Microbiol. 39: 403-406.

Zhelifonova, V. P., Antipova, T. V. and Kozlovsky, A. G. 2010. Secondary metabolites in taxonomy of the Penicillium fungi. Microbiology 79: 277-286. 